\title{
Primer reporte de Phoneutrismo en el Perú. Presentación de dos casos.
}

\author{
First case reports of phoneutrism in Peru. Presentation of two cases.
}

\author{
Maguiña Vargas Ciro ${ }^{1,2}$, Soto Arquíñigo Leslie ${ }^{2,3}$, Juárez Belaúnde Alan ${ }^{4}$, Magnifico Arfinengo Bruno ${ }^{4}$, Villón \\ Valenzuela Angie Candy ${ }^{5}$, Osores Plenge Fernando ${ }^{6}$
}

\section{RESUMEN}

Se presentan dos casos de mordedura de la araña Phoneutria spp. atendidos en el Hospital Nacional Cayetano Heredia (HNCH) en los años 2005 y 2008. En ambos casos la mordedura fue de sintomatología leve y de buena evolución, y se debió a un accidente laboral en personas que trabajan en mercados de venta de fruta al extraer plátanos de cajas procedentes de Tingo María (Selva central). El phoneutrismo es un accidente por arácnidos poco común; sin embargo, sería un problema importante de salud pública sobretodo en las zonas rurales en donde el comercio de plátanos es una de las principales fuente de ingresos de la población. El personal médico no conoce del todo a esta araña, así como la clínica de sus mordeduras, por ello presentamos estos casos que permitirán reconocer estos accidentes para su adecuado manejo y control. (Rev. Med Hered. 2008;19:128-133)

PALABRAS CLAVE: Phoneutria, Phoneutrismo, mordedura de araña.

\section{SUMMARY}

We present two cases of Phoneutria spp. spiderbite seen at the Hospital Nacional Cayetano Heredia (HNCH) in 2005 and 2008 respectively. In both cases, the bite had few symptoms with a good evolution, and occured as workrelated accidents in fruit market salespeople while extracting bananas from Tingo Maria (central peruvian jungle). Phoneutrism is a relatively common accidental bite; nevertheless it can become an important public health problem in the rural zones where banana trade is one of main income sources for the local population. Medical personnel in the area is mostly unaware of this spider or of the clinical picture its bite produces. We hope, by presenting these

\footnotetext{
${ }^{1}$ Médico Infectólogo Tropicalista y Dermatólogo. Instituto de Medicina Tropical Alexander von Humboldt”. Universidad Peruana Cayetano Heredia. Profesor de la Facultad de Medicina Alberto Hurtado, Universidad Peruana Cayetano Heredia. Lima, Perú.

${ }^{2}$ Departamento de Enfermedades Infecciosas Tropicales y Dermatológicas Hospital Nacional Cayetano Heredia. Lima, Perú.

${ }^{3}$ Médico Internista- Infectólogo, Instituto de Medicina Tropical Alexander von Humboldt. Universidad Peruana Cayetano Heredia. Lima, Perú. Médico Asistente del Departamento de Medicina del Hospital Nacional Cayetano Heredia. Lima, Perú.

${ }^{4}$ Medico Cirujano. Universidad Peruana Cayetano Heredia. Lima, Perú.

${ }^{5}$ Estudiante de Medicina. Universidad Peruana Cayetano Heredia. Lima, Perú.

${ }^{6}$ Médico Magister en Enfermedades Infecciosas y Tropicales. Profesor Facultad de Medicina de la Universidad Ricardo Palma. Lima, Perú.
} 
two cases, to allow them to recognize these accidents for its appropriate diagnosis, management and control. (Rev. Med Hered. 2008;19:128-133)

KEY WORDS: Phoneutria, phoneutrism, spiderbites.

\section{INTRODUCCIÓN}

El phoneutrismo es un accidente causado por la mordedura de la araña Phoneutria spp. Esta araña se encuentra localizada en toda Sudamérica (1-7), presenta varios géneros, siendo en nuestro país la Phoneutria nigriventer, la cual tiene reportada en otros países la peor casuística en cuanto a envenenamiento (2) y es la de mayor estudio por las características neurotóxicas de su veneno (8). Esta causa síntomas leves como dolor local, agitación y taquicardia, que es lo más común, hasta edema pulmonar y choque (7,9-12).

No existen casos reportados en nuestro país y no contamos con el suero antiveneno. Aunque los casos son aparentemente escasos, son arañas reconocidas en nuestro territorio y forman parte de las cuatro especies de arácnidos más venenosos según la OMS, por lo que es un importante problema de salud pública. El personal de salud debe reconocer las características del phoneutrismo y su adecuado tratamiento. A continuación presentamos dos casos reportados en el Hospital Nacional Cayetano Heredia (HNCH).

\section{Caso Clínico 1}

Mujer de 30 años de edad, natural del Cusco, procedente de Lima, comerciante de frutas. Una hora antes del ingreso al servicio de emergencia del $\mathrm{HNCH}$ mientras acomodaba una caja de plátanos procedente de Tingo María, sufre súbitamente dolor intenso en tercer dedo de mano derecha que se irradió hasta la base del miembro mencionado. La paciente identificó una araña de color café de aproximadamente $4 \mathrm{~cm}$. Inmediatamente presentó palpitaciones, diaforesis y aumento de volumen con cianosis del dedo afectado. Se decidió su hospitalización y se envió la araña para su identificación al Instituto Nacional de Salud (Figura $\mathrm{N}^{\circ} 1$ ).

Al examen físico se evidenció presión arterial 130/ 85 mmHg.; frecuencia cardiaca 80/min.; frecuencia respiratoria $16 / \mathrm{min}$; temperatura de $36.5^{\circ} \mathrm{C}$. En el tercer dedo de la mano derecha se observó dos lesiones puntiformes en la base ungueal y aumento de volumen

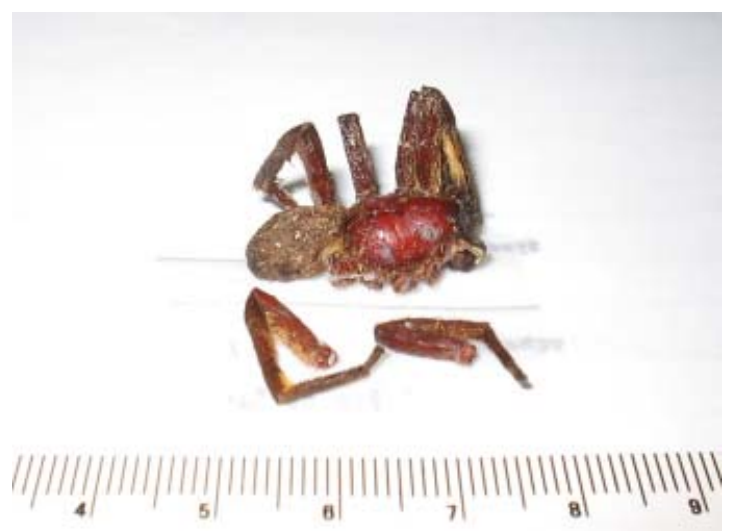

Figura $N^{\circ} 1$. Araña causante de la lesión de la paciente del primer caso. Género Phoneutria especie nigriventer según identificación del Instituto Nacional de Salud.

(Figura $\mathrm{N}^{\circ} 2$ ). El resto del examen no reveló alteración alguna.

Los exámenes auxiliares al ingreso fueron: hematocrito 38\%; leucocitos $8700 / \mathrm{mm}^{3}$, sin desviación izquierda, plaquetas 202 000/. $\mathrm{mm}^{3}$, el análisis de gases arteriales con $\mathrm{FiO}_{2}$ de 0,21 fue $\mathrm{PO}_{2}$ 80,9; pH: 7,37; $\mathrm{PCO}_{2} 39 ; \mathrm{HCO}_{3}: 22,3 \mathrm{mEq} / \mathrm{L}$; saturación arterial de $\mathrm{O}_{2}$ : 95\%, glucosa $79 \mathrm{mg} / \mathrm{dL}$; urea: $22 \mathrm{mg} / \mathrm{dL}$; creatinina: 0,68 mg/dL, magnesio: 1,7 mg/dl; calcio: 8,6 mg/dl; sodio: $141 \mathrm{mEq} / \mathrm{L}$; potasio: 3,64 mEq/L.; TGO: $22 \mathrm{U} /$

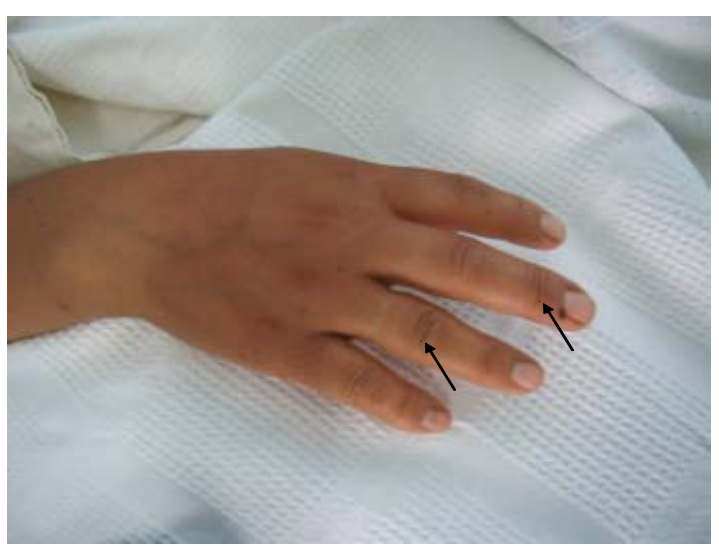

Figura $N^{\circ} 2$. Presencia de dos lesiones puntiformes en base ungueal y aumento de volumen de tercer dedo mano derecha de la paciente. 
L; TGP: 27 U/L; amilasa 57 U/L; DHL: 408 UI/L. La radiografía de tórax fue normal.

En la evolución, presentó elevación leve de presión arterial que disminuyó y se estabilizó durante la hospitalización. El síntoma más relevante fue el dolor intenso que fue manejado con tramadol horario mejorando significativamente, además recibió clindamicina.

La araña fue identificada en el Instituto Nacional de Salud como Phoneutria nigriventer.

\section{Caso Clínico 2}

Mujer de 55 años de edad, natural de Ancash, procedente de Lima, comerciante de frutas. Dos horas antes del ingreso al servicio de emergencia del $\mathrm{HNCH}$ mientras manipulaba una caja de plátanos procedente de Tingo María, sufre bruscamente dolor intenso tipo hincada en el tercer dedo de la mano derecha, y evidencia una araña de color café (Figura $\mathrm{N}^{\circ} 3$ ). Posteriormente, presenta aumento progresivo del volumen de mano y tercio distal de antebrazo así como cianosis del dedo afectado.

Al examen físico la presión arterial fue 110/70 mmHg.; frecuencia cardiaca 96/min.; frecuencia respiratoria $20 / \mathrm{min}$.; temperatura de $36^{\circ} \mathrm{C}$. En la piel de la base ungueal del tercer dedo de la mano derecha se observó una lesión puntiforme necrótica y aumento de volumen en la mano y tercio distal de antebrazo (Figura $\mathrm{N}^{\circ} 4$ ).

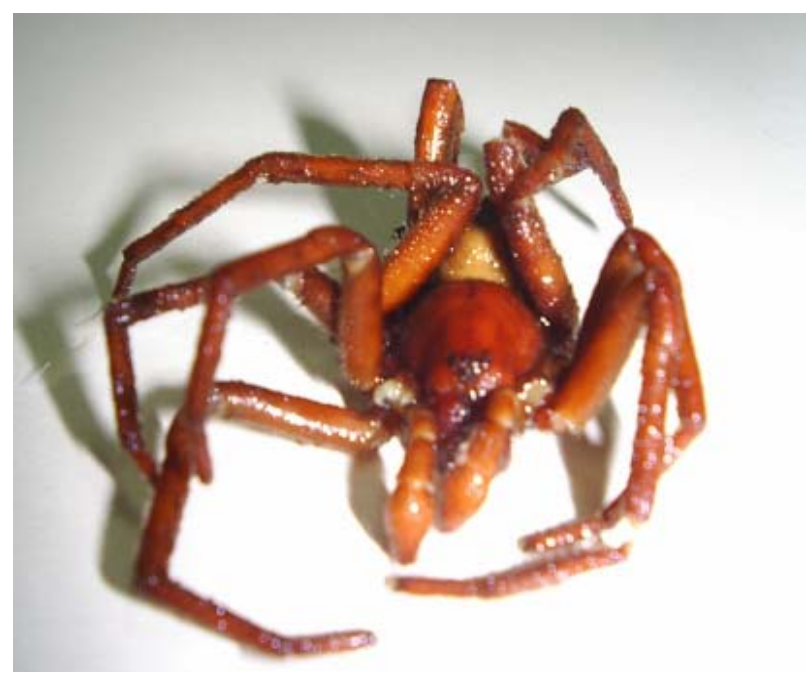

Figura $N^{\circ} 3$. Araña causante de la lesión de la paciente del segundo caso. Género Phoneutria.
Los exámenes auxiliares al ingreso fueron hematocrito $40 \%$; leucocitos $8,700 / \mathrm{mm}^{3}$, plaquetas $324,000 / \mathrm{mm}^{3}$, creatinina $0,5 \mathrm{mg} / \mathrm{dL}$; DHL $575 \mathrm{UI} / \mathrm{L}$;

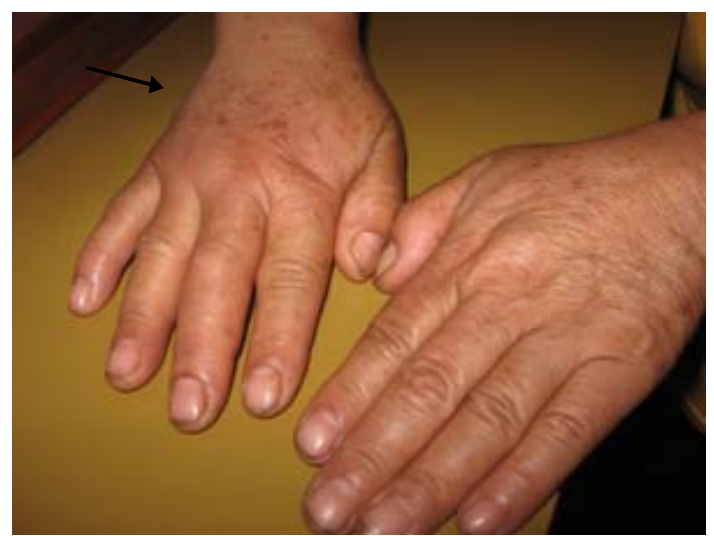

Figura $N^{\circ} 4$. Presencia de lesión puntiforme necrótica en base ungueal y aumento de volumen en mano.

bilirrubina total 0,4 mg/dL (directa: 0,2 mg/dL; indirecta: $0,2 \mathrm{mg} / \mathrm{dL}$ ).

En la evolución, la paciente no presentó síntomas ni signos vaso vagales. El síntoma más importante fue el dolor, que fue manejado con tramadol, mejorando significativamente. Además recibió clindamicina y dexametasona.

\section{DISCUSIÓN}

La Organización Mundial de la Salud (OMS) considera cuatro géneros de arañas de verdadero interés médico por las manifestaciones clínicas y la letalidad de sus venenos (Lactrodectus, Loxosceles, Phoneutria y Atrax) (1-3). El accidente aracnídico en América es un problema de salud pública en países como Chile, Estados Unidos y Brasil, siendo las mordeduras por Loxosceles spp. más frecuentes en los dos primeros países y por Phoneutria spp. en Brasil, en especial en zonas rurales $(2,4,5)$.

En el Perú se conoce muy pocos casos de mordedura por Phoneutria spp.y los conocimientos taxónomicos, toxicológicos y clínicos son escasos; pero se sabe de su presencia en zonas de la selva y debido al comercio de frutas que llega a la capital (Lima), el personal que labora en mercados la han reconocido en las cajas de estos alimentos como el plátano, como es el caso de las dos pacientes. Además en diciembre 2007 nos reportaron otro caso de un soldado mordido 
por la araña del genero Phoneutria, procedente de la Región Loreto, el cual presentó dolor intenso, edema en el hombro derecho, que fue evacuado a Iquitos, $\mathrm{y}$ tuvo una evolución favorable.

El phoneutrismo es causado por las arañas del género Phoneutria llamadas también arañas armadas o arañas de las bananeras y pertenecen a la familia Ctenidae (2,6). Se distribuyen ampliamente en América, sobre todo en Brasil, donde se presentan cerca de 4 000 casos de mordeduras de arañas, siendo el $60 \%$ de éstos por Phoneutria spp. $(6,7)$. En países de Europa y en Estados Unidos se han reportado casos, debido al viaje de las arañas en las cajas de bananos importadas desde Costa Rica y Colombia $(13,14)$.

Son arañas solitarias, ágiles, no tejen telas, frecuentes en cultivos, pero también penetran en las viviendas, refugiándose en los zapatos. Su cuerpo alcanza hasta 3,5 a $4,0 \mathrm{~cm}$. de longitud y con las extremidades extendidas hasta 15 - $18 \mathrm{~cm}$., el cuerpo está cubierto de pelos color gris marrón con manchas claras pareadas en el dorso dispuestas en filas longitudinales y oblicuas, y una fórmula ocular de 2,4,2 que lo diferencia de otros géneros $(2,15)$.

En América estas arañas se distribuyen desde Costa Rica hasta Brasil y Bolivia. Se han descrito seis especies: P. fere (Brasil, Ecuador, Perú y Las Guyanas); P. keyserlingi (Brasil); P. nigriventer (Brasil); P. reidy (Brasil), P. colombiana (Colombia) y P. boliviensis (Desde Costa Rica hasta Bolivia). De todas, la $P$. nigriventer es la más importante en relación con envenenamiento, siendo su veneno la más estudiada a la fecha $(2,7,8,16)$.

La mayoría de las mordeduras ocurre en los meses de marzo y abril, y menos en setiembre y octubre; mayormente afecta a hombres y ocurren durante el día, dentro de las casas o durante las actividades de trabajo. Los miembros (pies y manos) son los sitios anatómicos más comunes de las mordeduras (7).

La araña armada Phoneutria nigriventer tiene hábitos nocturnos y erráticos, caza y come una variedad de animales incluyendo muchas especies de insectos, otras arañas y pequeños roedores. Su veneno posee una diversidad de potentes enzimas y toxinas (histamina, serotonina, poliaminas, péptidos neurotóxicos y miotóxicos: PhTx 1,2,3 y 4), que bloquean la transmisión neuromuscular actuando sobre el receptor glutámico postsináptico y activando o inhibiendo diferentes canales de sodio, potasio y calcio en las terminaciones nerviosas motoras, sensitivas y autónomas. Produce liberación masiva de acetilcolina o catecolaminas que daría un disbalance adrenérgico/ colinérgico que causaría las manifestaciones severas del phoneutrismo; además de inducir extravasación de plasma mediado por activación del sistema kalicreinakininógeno-kinina que podría explicar los casos de edema pulmonar en accidentes graves $(2,8,17)$.

La mayoría de los pacientes que acuden por mordedura de Phoneutria spp. presenta dolor, edema, hipertermia, fasciculaciones y sudoración, siendo el dolor la manifestación clínica mas frecuente.

Los accidentes se clasifican como leve con dolor, taquicardia y agitación, como fue en los dos casos reportados; moderado, en el que aparece además sudoración profusa, vómitos ocasionales, dolor abdominal, sialorrea, priapismo e hipertensión y envenenamiento severo asociado además a bradicardia o taquicardia, hipertensión o hipotensión, arritmias, disnea, confusión o estupor, edema pulmonar o shock en casos raros (Tabla $\left.\mathrm{N}^{\circ} 1\right)(1,2,7,9-12)$.

Los pacientes pediátricos y adultos mayores de 70 años tienen una mayor frecuencia de los envenenamientos moderados comparados con el resto de población $(2,7,18)$.

Las alteraciones cardiacas como la arritmia y la taquicardia son frecuentes, pero no se observaron en nuestros pacientes. La hipertensión no es prevenida por el uso de propanolol o por antagonistas de receptores alfa-adrenérgicos como la fenoxibenzamina. Esto refleja que el veneno induce cambios hemodinámicas, no relacionados con la activación del sistema simpático, que produce la hipertensión (2,9). La hipotensión se presenta después de la hipertensión y parece estar mediada por la activación de canales de $\mathrm{K}^{+}$ATP

Tabla N $\mathbf{N}^{\mathbf{1}}$. Clasificación del envenenamiento por Phoneutria spp y tratamiento*.

\begin{tabular}{|c|c|c|c|}
\hline Intensidad & Local & Sistémico & Tratamiento \\
\hline $\begin{array}{l}\text { LEVE } \\
(84 \%-90 \%)\end{array}$ & $\begin{array}{l}\text { Dolor, edema, } \\
\text { sudoración, } \\
\text { fasciculaciones }\end{array}$ & Agitación y taquicardia & $\begin{array}{l}\text { Analgesia } \\
\text { observación 6-12 horas }\end{array}$ \\
\hline $\begin{array}{l}\text { MODERADO } \\
(9 \%-11 \%)\end{array}$ & Igual & $\begin{array}{l}\text { Además, vómito, dolor } \\
\text { abdominal, sialorrea, } \\
\text { sudoración profusa, } \\
\text { priapismo (niños), } \\
\text { hipertensión. }\end{array}$ & $\begin{array}{l}\text { Analgesia, } \\
\text { hospitalización, } \\
\text { antieméticos, } \\
\text { hidratación. } \\
\text { Suero antiaracnídico i.v. } \\
\text { (2 a } 4 \text { ampollas) }\end{array}$ \\
\hline $\begin{array}{l}\text { GRAVE } \\
(1 \%-5 \%)\end{array}$ & Igual & $\begin{array}{l}\text { Además, bradicardia o } \\
\text { taquicardia, hiper o } \\
\text { hipotensión, arritmias, } \\
\text { disnea, confusión o } \\
\text { estupor, edema } \\
\text { pulmonar o choque en } \\
\text { raros casos }\end{array}$ & $\begin{array}{l}\text { Analgesia, } \\
\text { hospitalización en la } \\
\text { UCI, antieméticos, } \\
\text { hidratación, } \mathrm{O}_{2} \text {, soporte } \\
\text { ventilatorio }(\dot{c}) \text {. } \\
\text { Suero antiaracnídico i.v. } \\
\text { (5 a } 10 \text { ampollas) }\end{array}$ \\
\hline
\end{tabular}


dependientes $(2,19)$. La bradicardia y la reducción del flujo coronario se observan sólo cuando la dosis de veneno es superior a $100 \mathrm{mcg}$, en ratones (9). El priapismo se debe al exceso de acetilcolina. La muerte es extremadamente rara y se debe al edema pulmonar $o$ al choque inducido por el veneno $(2,6)$.

Los procedimientos para calmar el dolor local se realizan con frecuencia (solo anestesia local con lidocaína al $2 \%$ sin epinefrina de $3 \mathrm{a} 4 \mathrm{ml}$ en adultos y $1 \mathrm{a} 2 \mathrm{ml}$ en niños, hasta tres dosis con intervalos de una hora); anestesia local más analgésicos orales o parenterales; o solo analgésicos. No se prescriben analgésicos en caso de que el accidente se clasifique como asintomático o si el dolor ha mejorado o si es considerado leve y tolerable.

En el cuadro moderado el dolor se trata en la misma forma que en los casos leves no tolerables más el uso de antieméticos (metoclopramida i.v., 0,2 a 0,5 mg/Kg de peso) y luego, oral cada 8 a 12 horas. Además, se debe suministrar 2 a 4 ampollas de suero antiaracnídico polivalente diluidas en solución salina y observar el paciente durante 24 horas.

Los pacientes graves deben estar con terapia de sostenimiento en la UCI y de ser necesario requerir de ventilación asistida; en estos últimos casos, en Brasil se ha utilizado hasta 5 a 10 ampollas de antiveneno $(2,7,18,20)$. En nuestros casos, por tratarse de cuadros leves fueron manejados únicamente con analgésicos por vía oral (tramadol), cobertura antibiótica con clindamicina, y en el segundo caso con dexametasona (21).

La terapia del antiveneno se utiliza en menos del $5 \%$ de los casos, principalmente en niños menores de 7 años y en los ancianos $(1,18)$.

El antiveneno se administra intravenosamente (20$25 \mathrm{ml}$.) en pacientes clasificados como severos y moderados, dentro de los 5-20 minutos sin la medicación anterior. Un mililitro del antiveneno neutraliza 7,5 dosis mortales mínimas de Phoneutria nigriventer. En el Perú, no contamos con el suero antiveneno de Phoneutria spp polivalente $(1,2,7)$.

En conclusión, se reporta por primera vez en nuestro país dos casos de phoneutrismo, ambos ocasionados por accidente laboral en mercados limeños por manipulación de plátanos procedentes de la zona de Tingo Maria. Las arañas fueron reconocidas como
Phoneutria nigriventer. Ambos casos fueron clasificados como leves y manejados con analgésicos y cobertura antibiótica. Se reconoce el phoneutrismo como un accidente que merece mayor conocimiento en nuestro medio para la mejora en cuanto a su atención por la presencia de casos en los últimos años.

\section{Correspondencia:}

\section{Ciro Maguiña Vargas}

Av. Honorio Delgado 430, Urb. Ingeniería San Martín de Porres.Lima, Perú.

Correo electrónico: cirom@upch.edu.pe

\section{REFERENCIAS BIBLIOGRÁFICAS}

1. Isbister G, Graudins A, White J, Warrell D. Antivenom treatment in arachnidism. J Toxicol Clin Toxicol 2003; 41(3): 291-300.

2. Quintana J, Otero R. Envenenamiento aracnídico en las Américas. MEDUNAB 2002; 5(13): 1-9.

3. Vetter RS, Isibister GK. Medical aspects of spider bites. Annu Rev Entomol 2008; 53: 409-29.

4. Junghanss T, Bodio M. Medically important venomous animals: biology, prevention, first aid, and clinical management. Clin Infect Dis 2006; 43(10): 1309-17.

5. Lucas S. Spiders in Brazil. Toxicon 1988; 26(9): 759-72.

6. White J, Cardoso JL, Fan HW. Clinical toxicology of spider bite. En: Meier J, White J. Handbook of clinical toxicology of animals venoms and poisons. 1st ed. Boca Raton: C.R.C. Press;, 1997.p. 259-329.

7. Bucaretchi F, Deus RC, Hyslop S, Madureira PR, de Capitani EM, Vieira RJ. A clinical-epidemiological study of bites by spiders of the genus Phoneutria. Rev Inst Med Trop Sao Paulo 2000; 42(1):17-21.

8. Gomez M, Kalapothakis E, Guatimosim C, Prado M. Phoneutria Nigriventer Venom. a cocktail of toxins that affect ion channels. Cell Mol Neurobiol 2002; 22: 579-588.

9. Costa SK, Moreno H Jr, Brain SD, De Nucci G, Antunes E. The effect of Phoneutria nigriventer (armed spider) venom on arterial blood pressure of anaesthetised rats. Eur J Pharmacol 1996; 298 (2): 113-20.

10. Costa SK, DE Nucci G, Antunes E, Brain SD. Phoneutria nigriventer spider venom induces oedema in rat skin by activation of capsaicin sensitive sensory nerves. Eur J Pharmacol 1997; 339(2-3): 223-6.

11. Bucaretchi F, Collares EF. Effect of Phoneutria nigriventer spider venom on gastric emptying in rats. Braz J Med Biol Res 1996; 29(2): 205-11.

12. Antunes E, Marangoni RA, Borges NC, Hyslop S, Fontana MD, De Nucci G. Effects of Phoneutria nigriventer venom on rabbit vascular smooth muscle. Braz J Med Biol Res 1993; 26(1): 81-91. 
13. Valderrama R. Arañas, escorpiones e himenópteros de interés médico. Tópicos Selectos de Infectología. Medellín: Universidad de Antioquia; 1997.p. 21-31.

14. Otero R. Envenenamiento por mordedura de arenas y ciempiés. En: Otero, R, Ángel R, García M. Primer Simposio Colombiano de Toxicología. Medellín: Ecográficas Limitada. 1998.p. 179-88.

15. Pettersson L. Phoneutria Nigriventer. Swedens Herpetological Society magazine 2005; 1: 17-21.

16. Valerio C. Sobre la presencia de Phoneutria boliviensis (F.O.P. Cambridge) (Araneae, Ctenidae) en Costa Rica. The Journal of Arachnology 1982; 11: 101-102.

17. Costa S, Brain S, Antunes E, De Nucci G, Docherty R. Phoneutria nigriventer spider venom activates $5-\mathrm{HT}_{4}$ receptors in rat-isolated vagus nerve. British Journal of Pharmacology 2003; 139: 59-64.

18. Isbister GK, Graudins A, White J, Warrell D. Antivenom treatment in arachnidism. J Toxicol Clin Toxicol 2003; 41(3): 291-300.

19. Costa SK, Hyslop S, Nathan LP, et al. Activation by Phoneutria nigriventer spider venom of autonomic nerve fibers in the isolated rat Herat. Eur J Pharmacol 1998; 363: 139-46.

20. Silva LM, Lages CP, Venuto T, et al. Primary culture of venom glands from the Brazilian armed spider, Phoneutria nigriventer (Araneae, Ctenidae). Toxicon 2008; 51(3): 428-434.

Recibido: 07/08/08

Aceptado para publicación: 22/09/08 